\title{
Assessment of Diversity in Physiological Characters of Advanced Breeding Lines of Rice (Oryza sativa $\mathrm{L}$ )
}

\author{
D. Dev Kumar ${ }^{1 *}$, D. Vishnu Vardhan Reddy ${ }^{1}$, P. Raghuveer Rao ${ }^{2}$, \\ M. Sheshu Madhav ${ }^{3}$, V. Gouri Shankar ${ }^{4}$ and B. Vishalakshi ${ }^{3}$
}

${ }^{1}$ Department of Crop Physiology, ${ }^{4}$ Department of Genetics and Plant Breeding, College of Agriculture, Rajendranagar

${ }^{2}$ Department of Plant Physiology, Indian Institute Rice Research, Rajendranagar

${ }^{3}$ Biotechnology Division, Indian Institute Rice Research, Rajendranagar

Prof. Jayashankar Telangana State Agricultural University (PJTSAU), Hyderabad-500 030, India

*Corresponding author

\section{A B S T R A C T}

The experimental field was laid in pooled RBD design replicated trice with 30 advanced breeding lines of rice, quality check BPT-5204 (SP-351, SP-352, SP-353, SP-354, SP-355,

\section{Keywords}

Advanced breeding Lines of rice, Leaf area index, Leaf area duration and Crop growth rate

Article Info

Accepted:

17 June 2019

Available Online:

10 July 2019
SP-356, SP-357, SP-358, SP-359, SP-360, SP-70, SP-72, SP-63, SP-61, SP-69, SP-55, SP80, SP-25, SP-13, IR-64, SP-03, SP-02, SP-34, SP-37, NDR-359, BPT-5204, SP-08, JAYA, SP-75 and SP-57). Physiological parameters were measured at periodical intervals active tillering, panicle initiation, physiological maturity and harvest viz., leaf area index, leaf area duration and crop growth rate. Significant difference was recorded among the advanced breeding lines for leaf area index in both the seasons as well as in pooled data. The genotype SP-08 and SP-72 showed maximum LAI, LAD and CGR compared to quality check BPT-5204. While, IR-64 showed minimum during the flowering stage in first season. In second season genotype SP-08 and SP-72 showed maximum value compared to quality check BPT-5204. While, IR-64 showed the minimum during active tillering stage. Pooled data revealed that the genotypes SP-08 and SP-72 recorded maximum LAI (4.04), LAD (61days) and CGR (24.64 $\mathrm{g} \mathrm{m}^{-2}$ day $^{-1}$ ) compared to quality check BPT-5204. While IR-64 recorded minimum respectively. The range of leaf area duration 45-77days.

\section{Introduction}

Rice (Oryza sativa L.) belongs to the family Germaine and sub family Oryzoidea. As a cereal grain, it is the most important staple food crops in the world. In Asia, more than two billion people are getting $60-70$ per cent of their energy requirement from rice and its derived products. Among the rice growing countries, India has the largest area (42.27 mha) and production (105.24 $\mathrm{m} \mathrm{t}$ ) next to China $(144 \mathrm{~m} \mathrm{t})$. With an average productivity 
of $2.49 \mathrm{t} \mathrm{ha}^{-1}$, which shows increase marginal is still well below the world's average yield of $4.36 \mathrm{t} \mathrm{ha}^{-1}$ (FAOSTAT, 2014). At the current population growth rate $(1.5 \%)$, rice requirement of India by 2025 would be around $125 \mathrm{~m} \mathrm{t}$ (Kumar et al., 2009). To safeguard and sustain the food security in India, it is quite important to increase the productivity of rice under limited resources, especially land and water. Hence, the major challenges have been produce more rice per unit amount of natural resource.

Low productivity of rice in India is a major concern for food and nutritional security of more than $60 \%$ population which is dependent on rice. The increasing scarcity of water for agriculture is becoming a major problem in many countries, particularly the leading rice-producing countries like China and India, where competition for freshwater and growing demands for other sectors are increasing in future (Choudhary et al., 2010).

Plant height and flowering time are two of the important traits that affect plant architecture. Efforts were made in this study to characterize morphologically the-EMSinduced dwarf and early flowering mutants of rice variety Nagina22 and to study their mode of inheritance. Nine true breeding mutants generated earlier by EMS treatment were analysed for differences in their phenotypic characteristics recorded according to the national guidelines for Distinctness, Uniformity and Stability (DUS) (Kulkarni et al., 2013).

\section{Materials and Methods}

\section{Growth parameters}

Growth parameters were calculated by using the following formulae described by Watson (1952) and Radford (1967).

\section{Leaf area measurement}

Leaf area was measured by using LI-3100 Leaf area meter (LICOR-Lincoln, Nebraska, USA). Five hills in the third row of every plot were uprooted and leaves are separated and area was measured. From the leaf area of these five hills LAI was calculated.

\section{Leaf Area Index (LAI)}

The LAI was worked out using the formula proposed by Yoshida et al., (1976).

$$
\text { LAI }=\frac{\text { LA x Number of leaves plant }{ }^{-1}}{\text { Land area occupied by the plant }}
$$

Where,

$\mathrm{L}=$ Length of 3rd leaf from the top $(\mathrm{cm})$

$\mathrm{W}=$ Maximum width of the same leaf $(\mathrm{cm})$

$\mathrm{K}=$ Correction factor $(0.75)$

\section{Leaf Area Duration (LAD)}

The LAD during the crop growth period was calculated.

$$
\mathrm{LAD}=\frac{\mathrm{LA}_{1}+\mathrm{LA}_{2}}{2} \mathrm{X}\left(\mathrm{t}_{2}-\mathrm{t}_{1}\right) \text { days. }
$$

Where,

$\mathrm{L}_{1}=$ Leaf Area at time $\mathrm{t}_{1}$ $\mathrm{L}_{2}=$ Leaf Area at time $\mathrm{t}_{2}$

Crop Growth Rate (CGR) $\left(\mathrm{g} \mathrm{m}^{-2} \mathrm{~d}^{-1}\right)$

$\mathrm{CGR}=\left(\mathrm{W}_{2}-\mathrm{W}_{1}\right) /\left(\mathrm{t}_{2}-\mathrm{t}_{1}\right) \times(1 / \mathrm{P}) \mathrm{g} \mathrm{m}^{-2} \mathrm{day}^{-1}$

Where $\mathrm{W}_{1}$ and $\mathrm{W}_{2}$ are total dry weight of plant at times $t_{1}$ and $t_{2}$ and $p$ is the land area. 


\section{Results and Discussion}

\section{Leaf Area Index (LAI)}

\section{Leaf Area Index (LAI) at active tillering stage}

In the present study all the 30 genotypes showed increased trend in the leaf area index at active tillering stage (Table 1 and Figure 1). Significant difference was recorded among the genotypes for leaf area index in both the seasons as well as in pooled data. The genotype SP-08 (3.23) and SP-72 (2.94) showed maximum LAI compared to quality check BPT-5204. While, IR-64 (1.30) showed minimum during in first season. In second season genotype SP-08 (3.40) and SP-72 (2.78) showed maximum value compared to quality check BPT-5204. While, IR-64 (1.60) showed the minimum during active tillering stage. Pooled data revealed that the genotype SP-08 (3.17) and SP-72 (2.71) recorded maximum LAI compared to quality check BPT-5204. While IR-64 recorded minimum (1.45) respectively.

\section{Leaf Area Index (LAI) at panicle initiation stage}

In the present study all the 30 genotypes showed increased trend in the leaf area index at panicle initiation stage (Table 1 and Figure 1). Significant difference was recorded among the genotypes for leaf area index in both the seasons as well as in pooled data. The genotype SP-08 (5.79) and SP-72 (5.42) showed maximum LAI compared to quality check BPT-5204. While, IR-64 (2.42) showed minimum during the in first season. In second season genotype SP-08 (5.82) and SP-72 (5.67) showed maximum value compared to quality check BPT-5204. While, IR-64 (2.95) showed the minimum during flowering stage. Pooled data revealed that the genotype SP-08 (5.81) and SP-72 (5.55) recorded maximum
LAI compared to quality check BPT-5204. While, IR-64 recorded minimum (2.69) respectively. In experiments of Azarpour et al., (2011) with increasing in nitrogen fertilizer rice LAI were raised. LAI trend in different rice cultivars is illustrated in Khazar late maturity cultivar (70 days after transplanting) had the highest LAI and after that Ali-Kazemi and Hashemi early-maturity cultivars (60 days after transplanting). One of the important growth indicators which have been being used as a photosynthetic system measurement is leaf area index (LAI). LAI is related to the biologic and economic yields and increase in LAI causes higher yield (Singh et al., 2009).

Leaf Area Index (LAI) at physiological maturity stage

In the present study all the 30 genotypes showed increased trend in the leaf area index at physiological maturity stage (Table 1 and depicted Figure 2). Significant difference was recorded among the genotypes for leaf area index in both the seasons as well as in pooled data. The genotype SP-08 (3.76) and SP-72 (3.40) showed maximum LAI compared to quality check BPT-5204. While, IR-64 (1.97) showed minimum during the first season. In second season genotype SP-08 (4.40) and SP72 (3.68) showed maximum value compared to quality check BPT-5204. While, IR-64 (1.89) showed the minimum during physiological maturity stage. Pooled data revealed that the genotypes SP-08 (4.08) and SP-72 (3.54) recorded maximum LAI compared to quality check BPT-5204. While, IR-64 recorded minimum (1.93) respectively.

\section{Leaf Area Index (LAI) at harvest stage}

In the present study all the 30 genotypes showed increased trend in the leaf area index at harvest stage (Table 1 and depicted Figure 2). Significant difference was recorded among 
the genotypes for leaf area index in both the seasons as well as in pooled data. The genotypes SP-08 (2.19) and SP-72 (2.00) showed maximum LAI compared to quality check BPT-5204. While, IR-64 (0.63) showed minimum during the first season. In second season genotype SP-08 (2.54) and SP-72 (2.03) showed maximum value compared to quality check BPT-5204. While, IR-64 (0.64) showed the minimum during flowering stage. Pooled data revealed that the genotype SP-08 (2.34) and SP-72 (2.01) recorded maximum LAI compared to quality check BPT-5204. While, IR-64 recorded minimum (0.33) respectively.

\section{Leaf Area Duration (LAD)}

It sounds that nitrogen shortage with reduction in vegetative growth and light use efficiency and as a result reduces in leaf area extension decreased LAD. Maximum LAD in each treatment was obtained about 65 days after sowing. LAD which shows the value of leaf area and photosynthetic tissues durability can be appropriate indicator of production. Correlation of LAD and grain yield is positive and so high and compared to the LAR has more correlation with grain yield (Sadeghi and Bohrani, 2001) since produced leaf area is important for the plant when it has capability to photosynthesis for a long time and a leaf which has not durability is not beneficial for the plant and a plant consumes more energy and photosynthesis assimilates for leaf production so the leaves which have longer life are more capable to compensate consumed photosynthesis assimilates for its production.

\section{Leaf Area Duration (LAD) at active tillering stage}

Data on the 30 genotypes showed that significant difference on the genotypes leaf area duration at active tillering stage (Table 2 and depicted Figure 3). The genotypes SP-08 (59days) and SP-72 (56days) showed maximum leaf area duration compared to quality check BPT-5204 respectively while, minimum leaf area duration was recorded by IR-64 (23days) respectively in first season. The genotypes SP-08 (57days) and SP-72 (55days) recorded maximum values compared to quality check BPT-5204. While, IR- 64 (29days) recorded minimum values respectively during second season. Pooled data revealed that the genotypes SP-08 (58days) and SP-72 (55days) recorded maximum leaf area duration compared to quality check BPT-5204. While, IR-64 (26days) recorded minimum value respectively.

\section{Leaf Area Duration (LAD) at panicle initiation stage}

Data on the 30 genotypes showed that significant difference on the genotypes leaf area duration at panicle initiation stage (Table 2 and depicted Figure 3). The genotype SP-08 (78days) and SP-72 (75days) showed maximum leaf area duration compared to quality check BPT-5204 respectively while minimum leaf area duration was recorded by and IR-64 (42days) respectively in first season. The genotypes SP-08 (76days) and SP-72 (74days) recorded maximum values while, IR- 64 (48days) recorded minimum value respectively during second season. Pooled data revealed that the genotypes SP-08 (77days) and SP-72 (74days) recorded maximum leaf area duration while, IR-64 (45days) recorded minimum value respectively.

\section{Leaf Area Duration (LAD) at physiological maturity stage}

Data on the 30 genotypes showed that significant difference on the genotypes leaf area duration at physiological maturity stage 
(Table 2 and depicted Figure 4). The genotype SP-08 (66days) and SP-72 (63days) showed maximum leaf area duration compared to quality check BPT-5204 respectively while, minimum leaf area duration was recorded by IR-64 (30days) respectively in first season. The genotypes SP-08 (64days) and SP-72 (62days) recorded maximum values compared to quality check BPT-5204. While, IR- 64 (36days) recorded minimum values respectively during second season. Pooled data revealed that the genotypes SP-08 (65days) and SP-72 (62days) recorded maximum leaf area duration compared to quality check BPT5204. While, IR-64 (33days) recorded minimum value respectively.

\section{Leaf Area Duration (LAD) at harvest stage}

Data on the 30 genotypes showed that significant difference on the genotypes leaf area duration at harvest stage (Table 2 and depicted Figure 4). The genotype SP-08 (52days) and SP-72 (50days) showed maximum leaf area duration respectively while, minimum leaf area duration was recorded compared by BPT-5204. IR-64 (16days) respectively in first season. The genotypes SP-08 (50days) and SP-72 (49days) recorded maximum values compared to quality check BPT-5204. While, IR- 64 (22days) recorded minimum value respectively during second season. Pooled data revealed that the genotypes SP-08 (51days) and SP-72 (49days) recorded maximum leaf area duration compared to quality check BPT-5204. While, IR-64 (19days) recorded minimum value respectively.

\section{Crop Growth Rate (CGR) $\mathrm{g} \mathrm{m}^{-2}$ day $^{-1}$}

The phenomena of CGR, RGR, NAR and PR tend to be low again during later stage and negative towards maturity considerably due to several reasons like leaves shading owing to early closure of canopy which hinder solar radiation absorbed by the leaves therefore, less photosynthetic assimilates produced which causes lowering the net assimilation rate, excessive leaf senescence after reproductive stage diminishing photosynthesis rate. These results are in agreement with those obtained by Paul et al., (2016).

Crop Growth Rate (CGR) $\mathrm{g} \mathrm{m}^{-2} \mathrm{day}^{-1}$ at active tillering

In the present study all the 30 genotypes showed increased trend in the crop growth rate at active tillering stage (Table 3 and depicted Figure 5). Significant difference was recorded among the genotypes for crop growth rate in both the seasons as well as in pooled data. The genotype SP-08 (12.17 $\mathrm{g} \mathrm{m}^{-2}$ day $^{-1}$ ) and SP-72 (10.77 $\mathrm{g} \mathrm{m}^{-2}$ day $\left.^{-1}\right)$ showed maximum crop growth rate compared to quality check BPT-5204. While, IR-64 (8.23 $\mathrm{g} \mathrm{m}^{-2}$ day $^{-1}$ ) showed minimum during the active tillering stage in first season. In second season genotype SP-08 (12.47 $\mathrm{g} \mathrm{m}^{-2}$ day $\left.^{-1}\right)$ and SP-72 (11.00 $\mathrm{g} \mathrm{m}^{-2}$ day $\left.^{-1}\right)$ showed maximum value compared to quality check BPT-5204. While, IR-64 (8.50 $\mathrm{g} \mathrm{m}^{-2}$ day $^{-1}$ ) showed the minimum value. Pooled data revealed that the genotype SP-08 $\left(12.32 \mathrm{~g} \mathrm{~m}^{-2}\right.$ day $^{-1}$ ) and SP-72 (10.90 $\mathrm{g} \mathrm{m}^{-2}$ day $\left.^{-1}\right)$ recorded maximum crop growth rate compared to quality check BPT-5204. While, IR-64 recorded minimum crop growth rate $(8.37 \mathrm{~g}$ $\mathrm{m}^{-2}$ day $^{-1}$ ) respectively.

\section{Crop Growth Rate (CGR) $\mathrm{g} \mathrm{m}^{-2}$ day $^{-1}$ at panicle initiation stage}

In the present study all the 30 genotypes showed increased trend in the crop growth rate at panicle initiation stage (Table 3 and depicted Figure 5). Significant difference was recorded among the genotypes for crop growth rate in both the seasons as well as in pooled data. 
Int.J.Curr.Microbiol.App.Sci (2019) 8(7): 2286-2298

Table.1 Leaf Area Index (LAI) at active tillering, panicle initiation, physiological maturity and harvest 2014, 2015 and pooled of advanced breeding lines of rice

\begin{tabular}{|c|c|c|c|c|c|c|c|c|c|c|c|c|c|}
\hline \multicolumn{14}{|c|}{ Leaf Area Index (LAI) } \\
\hline \multirow[b]{2}{*}{ S.No } & \multirow[b]{2}{*}{ Genotypes } & \multicolumn{3}{|c|}{ Active Tillering } & \multicolumn{3}{|c|}{ Panicle Initiation } & \multicolumn{3}{|c|}{ Physiological Maturity } & \multicolumn{3}{|c|}{ Harvest } \\
\hline & & 2014 & 2015 & Pooled & 2014 & 2015 & Pooled & 2014 & 2015 & Pooled & 2014 & 2015 & Pooled \\
\hline 1 & SP-351 & 1.86 & 1.94 & 1.70 & 2.81 & 3.77 & 3.29 & 2.73 & 2.89 & 2.81 & 1.24 & 1.27 & 1.26 \\
\hline 2 & SP-352 & 2.06 & 2.7 & 2.38 & 3.51 & 4.41 & 3.96 & 2.97 & 3.55 & 3.26 & 1.45 & 1.78 & 1.62 \\
\hline 3 & SP-353 & 1.83 & 2.01 & 1.92 & 3.27 & 3.54 & 3.41 & 2.92 & 3.26 & 3.09 & 1.29 & 1.33 & 1.31 \\
\hline 4 & SP354 & 1.52 & 1.74 & 1.63 & 4.57 & 5.35 & 4.96 & 2.63 & 3.34 & 2.99 & 1.28 & 1.31 & 1.30 \\
\hline 5 & SP-355 & 1.81 & 1.88 & 1.84 & 4.24 & 3.98 & 4.11 & 2.76 & 3.29 & 2.93 & 1.06 & 0.55 & 0.81 \\
\hline 6 & SP-356 & 2.56 & 2.36 & 2.46 & 4.94 & 5.43 & 5.19 & 2.76 & 3.00 & 2.88 & 1.63 & 1.67 & 1.65 \\
\hline 7 & SP-357 & 1.67 & 2.17 & 1.92 & 3.79 & 4.06 & 3.93 & 3.11 & 3.61 & 3.26 & 1.23 & 0.45 & 0.84 \\
\hline 8 & SP-358 & 1.4 & 1.99 & 1.70 & 4.73 & 5.73 & 5.23 & 2.54 & 3.09 & 3.43 & 1.40 & 0.44 & 0.92 \\
\hline 9 & SP-359 & 1.62 & 1.71 & 1.67 & 3.25 & 3.72 & 3.49 & 2.61 & 3.22 & 2.92 & 1.56 & 0.49 & 1.03 \\
\hline 10 & SP-360 & 1.8 & 2.01 & 1.91 & 3.64 & 5.32 & 4.48 & 3.1 & 3.22 & 3.16 & 1.73 & 0.43 & 1.08 \\
\hline 11 & SP-70 & 1.9 & 2.5 & 2.2 & 3.31 & 4.40 & 3.86 & 2.74 & 3.15 & 2.95 & 1.20 & 1.50 & 1.35 \\
\hline 12 & SP-72 & 2.94 & 2.78 & 2.71 & 5.42 & 5.67 & 5.55 & 3.40 & 3.68 & 3.54 & 2.00 & 2.03 & 2.01 \\
\hline 13 & SP-63 & 2.23 & 2.42 & 2.33 & 4.34 & 4.04 & 4.19 & 2.91 & 3.01 & 2.96 & 1.42 & 1.71 & 1.57 \\
\hline 14 & SP-61 & 2.4 & 2.47 & 2.44 & 3.92 & 4.07 & 4.00 & 3.11 & 3.42 & 3.27 & 1.82 & 1.72 & 1.77 \\
\hline 15 & SP-69 & 2.56 & 2.43 & 2.5 & 5.27 & 5.58 & 5.43 & 3.14 & 3.4 & 3.27 & 1.46 & 1.96 & 1.71 \\
\hline 16 & SP-55 & 2.73 & 2.28 & 2.51 & 2.76 & 3.86 & 3.31 & 2.91 & 3.41 & 3.16 & 1.42 & 1.55 & 1.49 \\
\hline 17 & SP-80 & 2.90 & 2.52 & 2.71 & 2.90 & 2.95 & 2.93 & 3.12 & 3.36 & 3.24 & 1.35 & 1.77 & 1.56 \\
\hline 18 & SP-25 & 3.06 & 2.4 & 2.73 & 4.99 & 5.15 & 5.07 & 2.71 & 3.14 & 2.93 & 1.90 & 1.95 & 1.93 \\
\hline 19 & SP-13 & 2.40 & 2.13 & 2.27 & 2.60 & 3.33 & 2.97 & 3.14 & 2.71 & 2.93 & 1.62 & 1.53 & 1.58 \\
\hline 20 & IR-64 & 1.30 & 1.60 & 1.45 & 2.42 & 2.95 & 2.69 & 1.97 & 1.89 & 1.93 & 0.23 & 0.42 & 0.33 \\
\hline 21 & SP-03 & 2.56 & 2.50 & 2.53 & 3.41 & 3.84 & 3.63 & 2.85 & 3.58 & 3.22 & 1.96 & 2.09 & 2.03 \\
\hline 22 & SP-02 & 2.73 & 2.40 & 2.57 & 2.96 & 3.67 & 3.32 & 2.16 & 2.77 & 2.47 & 1.90 & 2.00 & 1.95 \\
\hline 23 & SP-34 & 2.90 & 2.53 & 2.72 & 4.68 & 5.51 & 5.10 & 2.38 & 2.87 & 2.63 & 1.94 & 2.01 & 1.95 \\
\hline 24 & SP-37 & 2.06 & 2.41 & 2.24 & 3.87 & 5.25 & 4.56 & 2.82 & 3.89 & 3.36 & 0.90 & 0.78 & 0.84 \\
\hline 25 & NDR-359 & 2.23 & 2.25 & 2.24 & 2.76 & 3.62 & 3.19 & 2.6 & 2.59 & 2.6 & 0.56 & 0.66 & 0.61 \\
\hline 26 & ВРТ-5204 & 1.5 & 1.63 & 1.57 & 2.56 & 3.09 & 2.83 & 2.14 & 2.44 & 2.29 & 0.40 & 0.48 & 0.44 \\
\hline 27 & SP-08 & 3.23 & 3.4 & 3.17 & 5.79 & 5.82 & 5.81 & 3.76 & 4.40 & 4.08 & 2.19 & 2.54 & 2.34 \\
\hline 28 & JAYA & 2.73 & 2.33 & 2.53 & 3.16 & 4.03 & 3.60 & 2.88 & 2.54 & 2.71 & 0.73 & 0.56 & 0.65 \\
\hline 29 & SP-75 & 2.90 & 2.56 & 2.73 & 2.74 & 4.30 & 3.52 & 2.53 & 3.25 & 2.89 & 1.44 & 1.49 & 1.47 \\
\hline \multirow[t]{5}{*}{30} & SP-57 & 2.06 & 2.52 & 2.29 & 3.59 & 3.68 & 3.64 & 2.3 & 2.99 & 2.65 & 1.52 & 1.57 & 1.55 \\
\hline & Mean & 2.21 & 2.25 & 2.23 & 3.74 & 4.34 & 4.04 & 2.75 & 3.27 & 3.01 & 1.39 & 1.33 & 1.36 \\
\hline & $\mathrm{SE}(\mathrm{m})$ & 0.06 & 0.05 & 0.04 & 0.04 & 0.03 & 0.02 & 0.01 & 0.02 & 0.01 & 0.02 & 0.01 & 0.09 \\
\hline & $\mathrm{CD}$ at $5 \%$ & 1.19 & 1.22 & 1.33 & 1.09 & 1.08 & 1.06 & 1.03 & 1.04 & 1.02 & 1.00 & 1.00 & 1.02 \\
\hline & $\mathrm{CV}$ & 0.61 & 4.12 & 5.75 & 1.92 & 2.18 & 1.71 & 0.46 & 0.52 & 0.34 & 0.10 & 0.82 & 0.51 \\
\hline
\end{tabular}


Table.2 Leaf Area Duration (days) at active tillering, panicle initiation, physiological maturity and harvest 2014, 2015 and pooled of advanced breeding lines of rice

\begin{tabular}{|c|c|c|c|c|c|c|c|c|c|c|c|c|c|}
\hline & \multicolumn{13}{|c|}{ Leaf Area Duration (LAD) } \\
\hline & \multicolumn{4}{|c|}{ Active Tillering } & \multicolumn{3}{|c|}{ Panicle Initiation } & \multicolumn{3}{|c|}{$\begin{array}{c}\text { Physiological } \\
\text { Maturity }\end{array}$} & \multicolumn{3}{|c|}{ Harvest } \\
\hline $\begin{array}{c}\text { S. } \\
\text { No }\end{array}$ & Genotypes & 2014 & 2015 & Pooled & 2014 & 2015 & Pooled & 2014 & 2015 & Pooled & 2014 & 2015 & Pooled \\
\hline 1 & SP-351 & 37 & 42 & 39 & 56 & 61 & 59 & 44 & 49 & 47 & 30 & 35 & 32 \\
\hline 2 & SP-352 & 40 & 38 & 39 & 59 & 57 & 58 & 47 & 45 & 46 & 33 & 31 & 32 \\
\hline 3 & SP-353 & 50 & 53 & 51 & 69 & 72 & 71 & 57 & 60 & 59 & 43 & 46 & 44 \\
\hline 4 & SP354 & 34 & 49 & 41 & 53 & 68 & 61 & 41 & 56 & 49 & 27 & 42 & 34 \\
\hline 5 & SP-355 & 33 & 40 & 36 & 52 & 59 & 56 & 40 & 47 & 44 & 26 & 33 & 29 \\
\hline 6 & SP-356 & 33 & 46 & 39 & 52 & 65 & 59 & 40 & 53 & 47 & 26 & 39 & 32 \\
\hline 7 & SP-357 & 45 & 50 & 47 & 64 & 69 & 67 & 52 & 57 & 55 & 38 & 43 & 40 \\
\hline 8 & SP-358 & 34 & 42 & 38 & 53 & 61 & 57 & 41 & 49 & 45 & 27 & 35 & 31 \\
\hline 9 & SP-359 & 35 & 47 & 41 & 54 & 66 & 60 & 42 & 54 & 48 & 28 & 40 & 34 \\
\hline 10 & SP-360 & 48 & 53 & 50 & 67 & 72 & 70 & 55 & 60 & 58 & 41 & 46 & 43 \\
\hline 11 & SP-70 & 32 & 43 & 37 & 51 & 62 & 57 & 39 & 50 & 45 & 25 & 36 & 30 \\
\hline 12 & SP-72 & 56 & 55 & 55 & 75 & 74 & 74 & 63 & 62 & 62 & 50 & 49 & 49 \\
\hline 13 & SP-63 & 38 & 45 & 41 & 57 & 64 & 61 & 45 & 52 & 49 & 31 & 38 & 34 \\
\hline 14 & SP-61 & 55 & 50 & 52 & 74 & 69 & 72 & 62 & 57 & 60 & 48 & 43 & 45 \\
\hline 15 & SP-69 & 42 & 54 & 48 & 61 & 73 & 67 & 49 & 61 & 55 & 35 & 47 & 41 \\
\hline 16 & SP-55 & 43 & 45 & 44 & 62 & 64 & 63 & 50 & 52 & 51 & 36 & 38 & 37 \\
\hline 17 & SP-80 & 39 & 50 & 44 & 58 & 69 & 64 & 46 & 57 & 52 & 32 & 43 & 37 \\
\hline 18 & SP-25 & 52 & 51 & 51 & 71 & 70 & 71 & 59 & 58 & 59 & 45 & 44 & 44 \\
\hline 19 & SP-13 & 45 & 39 & 42 & 64 & 58 & 61 & 52 & 46 & 49 & 38 & 32 & 35 \\
\hline 20 & IR-64 & 23 & 29 & 26 & 42 & 48 & 45 & 30 & 36 & 33 & 16 & 22 & 19 \\
\hline 21 & SP-03 & 49 & 52 & 50 & 68 & 71 & 70 & 56 & 59 & 58 & 42 & 45 & 43 \\
\hline 22 & SP-02 & 41 & 49 & 45 & 60 & 68 & 64 & 48 & 56 & 52 & 34 & 42 & 38 \\
\hline 23 & SP-34 & 44 & 44 & 44 & 63 & 63 & 63 & 51 & 51 & 51 & 37 & 37 & 37 \\
\hline 24 & SP-37 & 41 & 51 & 46 & 60 & 70 & 65 & 48 & 58 & 53 & 34 & 44 & 39 \\
\hline 25 & NDR-359 & 33 & 34 & 33 & 52 & 53 & 53 & 40 & 41 & 41 & 26 & 27 & 26 \\
\hline 26 & ВРT-5204 & 19 & 25 & 22 & 38 & 44 & 41 & 26 & 32 & 29 & 12 & 18 & 15 \\
\hline 27 & SP-08 & 59 & 57 & 58 & 78 & 76 & 77 & 66 & 64 & 65 & 52 & 50 & 51 \\
\hline 28 & JAYA & 34 & 37 & 35 & 53 & 56 & 55 & 41 & 44 & 43 & 27 & 30 & 28 \\
\hline 29 & SP-75 & 35 & 45 & 38 & 56 & 64 & 59 & 38 & 52 & 45 & 24 & 38 & 31 \\
\hline 30 & SP-57 & 34 & 42 & 38 & 53 & 61 & 57 & 41 & 49 & 45 & 27 & 35 & 31 \\
\hline & Mean & 39 & 45 & 42 & 59 & 64 & 61 & 47 & 52 & 49 & 33 & 38 & 35 \\
\hline & $\mathrm{SE}(\mathrm{m})$ & 0.008 & 0.010 & 0.005 & 0.006 & 0.004 & 0.002 & 0.023 & 0.001 & 0.019 & 0.025 & 0.038 & 0.027 \\
\hline & $\mathrm{CD}$ at $5 \%$ & 1.016 & 1.019 & 1.014 & 2.004 & 2.006 & 1.005 & 1.001 & 1.002 & 1.055 & 1.065 & 1.054 & 1.020 \\
\hline & $\mathrm{CV}$ & 3.088 & 4.281 & 2.356 & 4.170 & 5.560 & 3.449 & 3.450 & 2.780 & 2.950 & 1.950 & 1.820 & 1.164 \\
\hline
\end{tabular}


Table.3 Crop Growth Rate (CGR) $\mathrm{g} \mathrm{m}^{-2}$ day $^{-1}$ at active tillering, panicle initiation and physiological maturity and harvest 2014, 2015 and pooled of advanced breeding lines of rice

\begin{tabular}{|c|c|c|c|c|c|c|c|c|c|c|c|c|c|}
\hline \multicolumn{14}{|c|}{ Crop Growth Rate (CGR) g m ${ }^{-2}$ day $^{-1}$} \\
\hline & & \multicolumn{3}{|c|}{ Active tillering } & \multicolumn{3}{|c|}{ Panicle initiation } & \multicolumn{3}{|c|}{$\begin{array}{l}\text { Physiological } \\
\text { maturity }\end{array}$} & \multicolumn{3}{|c|}{ Harvest } \\
\hline S. No & Genotypes & 2014 & 2015 & Pooled & 2014 & 2015 & Pooled & 2014 & 2015 & Pooled & 2014 & 2015 & Pooled \\
\hline 1 & SP-351 & 9.63 & 9.93 & 9.78 & 25.70 & 25.67 & 25.69 & 14.43 & 14.73 & 14.58 & 5.97 & 5.90 & 5.94 \\
\hline 2 & SP-352 & 9.13 & 9.40 & 9.27 & 25.47 & 25.40 & 25.44 & 13.93 & 14.20 & 14.07 & 5.47 & 5.43 & 5.45 \\
\hline 3 & SP-353 & 10.03 & 10.30 & 10.17 & 27.10 & 27.03 & 27.07 & 14.83 & 15.10 & 14.97 & 6.40 & 6.33 & 6.37 \\
\hline 4 & SP354 & 8.57 & 8.90 & 8.74 & 23.73 & 23.67 & 23.70 & 13.37 & 13.70 & 13.54 & 4.90 & 4.80 & 4.85 \\
\hline 5 & SP-355 & 10.47 & 10.73 & 10.60 & 27.03 & 27.00 & 27.02 & 15.27 & 15.53 & 15.40 & 6.77 & 6.73 & 6.75 \\
\hline 6 & SP-356 & 10.63 & 10.90 & 10.77 & 28.17 & 28.10 & 28.14 & 15.43 & 15.70 & 15.57 & 6.93 & 6.90 & 6.92 \\
\hline 7 & SP-357 & 9.53 & 9.80 & 9.67 & 25.73 & 25.67 & 25.70 & 14.33 & 14.60 & 14.47 & 5.87 & 5.83 & 5.85 \\
\hline 8 & SP-358 & 9.50 & 9.80 & 9.65 & 25.53 & 25.47 & 25.50 & 14.30 & 14.60 & 14.45 & 5.87 & 5.77 & 5.82 \\
\hline 9 & SP-359 & 9.47 & 9.73 & 9.60 & 22.40 & 22.33 & 22.37 & 14.27 & 14.53 & 14.40 & 5.77 & 5.73 & 5.75 \\
\hline 10 & SP-360 & 9.27 & 9.57 & 9.42 & 22.57 & 22.50 & 22.54 & 14.07 & 14.37 & 14.22 & 5.63 & 5.57 & 5.60 \\
\hline 11 & SP-70 & 10.30 & 10.60 & 10.45 & 24.33 & 24.27 & 24.30 & 15.10 & 15.40 & 15.25 & 6.67 & 6.57 & 6.62 \\
\hline 12 & SP-72 & 10.77 & 11.03 & 10.90 & 29.20 & 29.17 & 29.19 & 15.57 & 15.83 & 15.70 & 7.07 & 7.03 & 7.05 \\
\hline 13 & SP-63 & 9.97 & 10.23 & 10.10 & 24.50 & 24.43 & 24.47 & 14.77 & 15.03 & 14.90 & 6.27 & 6.23 & 6.25 \\
\hline 14 & SP-61 & 9.53 & 9.83 & 9.68 & 22.83 & 22.77 & 22.80 & 14.33 & 14.63 & 14.48 & 5.90 & 5.80 & 5.85 \\
\hline 15 & SP-69 & 9.87 & 10.13 & 10.00 & 21.77 & 21.70 & 21.74 & 14.67 & 14.93 & 14.80 & 6.17 & 6.13 & 6.15 \\
\hline 16 & SP-55 & 9.43 & 9.73 & 9.58 & 23.33 & 23.27 & 23.30 & 14.23 & 14.53 & 14.38 & 5.77 & 5.70 & 5.74 \\
\hline 17 & SP-80 & 9.63 & 9.90 & 9.77 & 23.00 & 22.97 & 22.99 & 14.43 & 14.70 & 14.57 & 5.93 & 5.90 & 5.92 \\
\hline 18 & SP-25 & 9.57 & 9.87 & 9.72 & 21.60 & 21.53 & 21.57 & 14.37 & 14.67 & 14.52 & 5.93 & 5.83 & 5.88 \\
\hline 19 & SP-13 & 10.47 & 10.73 & 10.60 & 23.97 & 23.90 & 23.94 & 15.27 & 15.53 & 15.40 & 6.77 & 6.73 & 6.75 \\
\hline 20 & IR-64 & 8.23 & 8.50 & 8.37 & 17.13 & 17.10 & 17.12 & 13.03 & 13.30 & 13.17 & 4.53 & 4.47 & 4.50 \\
\hline 21 & SP-03 & 10.17 & 10.43 & 10.30 & 27.43 & 27.37 & 27.40 & 14.97 & 15.23 & 15.10 & 6.47 & 6.43 & 6.45 \\
\hline 22 & SP-02 & 9.30 & 9.57 & 9.44 & 25.60 & 25.53 & 25.57 & 14.10 & 14.37 & 14.24 & 5.63 & 5.60 & 5.62 \\
\hline 23 & SP-34 & 9.67 & 9.93 & 9.80 & 29.17 & 29.13 & 29.15 & 14.47 & 14.73 & 14.60 & 5.97 & 5.93 & 5.95 \\
\hline 24 & SP-37 & 10.73 & 11.00 & 10.87 & 28.83 & 28.77 & 28.80 & 15.53 & 15.80 & 15.67 & 7.02 & 7.00 & 7.01 \\
\hline 25 & NDR-359 & 8.73 & 9.00 & 8.87 & 22.43 & 22.37 & 22.40 & 13.53 & 13.80 & 13.67 & 5.03 & 5.00 & 5.02 \\
\hline 26 & BPT-5204 & 8.37 & 8.63 & 8.50 & 18.33 & 18.27 & 18.30 & 13.17 & 13.43 & 13.30 & 4.67 & 4.63 & 4.65 \\
\hline 27 & SP-08 & 12.17 & 12.47 & 12.32 & 32.00 & 31.93 & 31.97 & 16.97 & 17.27 & 17.12 & 8.53 & 8.43 & 8.48 \\
\hline 28 & JAYA & 8.4 & 8.7 & 8.55 & 20.87 & 20.83 & 20.85 & 13.20 & 13.50 & 13.35 & 4.77 & 4.67 & 4.72 \\
\hline 29 & SP-75 & 10.07 & 10.33 & 10.20 & 27.17 & 27.10 & 27.14 & 14.87 & 15.13 & 15.00 & 6.43 & 6.37 & 6.40 \\
\hline 30 & SP-57 & 9.8 & 10.1 & 9.95 & 23.27 & 23.20 & 23.24 & 14.60 & 14.90 & 14.75 & 6.17 & 6.07 & 6.12 \\
\hline & Mean & 9.71 & 9.99 & 9.85 & 24.67 & 24.62 & 24.64 & 14.51 & 14.79 & 14.65 & 6.22 & 5.68 & 5.84 \\
\hline & $\mathrm{SE}(\mathrm{m})$ & 0.019 & 0.016 & 0.029 & 0.009 & 0.008 & 0.028 & 0.010 & 0.016 & 0.386 & 0.045 & 0.028 & 0.038 \\
\hline & $\mathrm{CD}$ at $5 \%$ & 0.089 & 0.046 & 0.082 & 0.026 & 0.023 & 0.080 & 0.029 & 0.046 & 1.083 & 0.089 & 0.241 & 0.108 \\
\hline & $\mathrm{CV}$ & 0.372 & 0.214 & 0.827 & 0.048 & 0.293 & 0.333 & 0.072 & 0.114 & 2.713 & 0.890 & 0.920 & 0.671 \\
\hline
\end{tabular}


Figure.1 Leaf Area Index (LAI) at active tillering and panicle initiation 2014, 15 and pooled of advanced breeding lines of rice
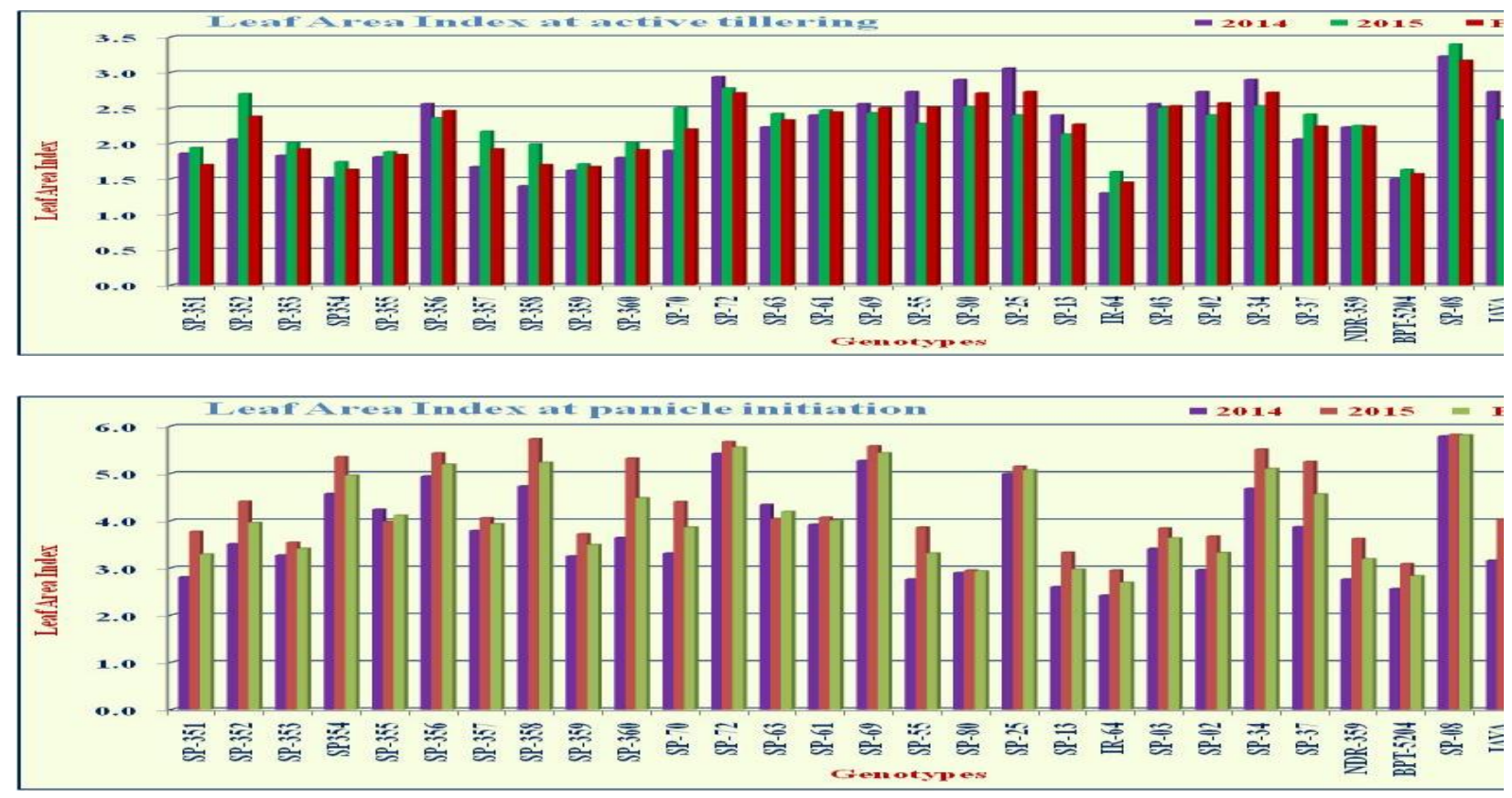

Figure.2 Leaf Area Index at active (LAI) physiological maturity and harvest 2014, 15 and pooled of advanced breeding lines of rice

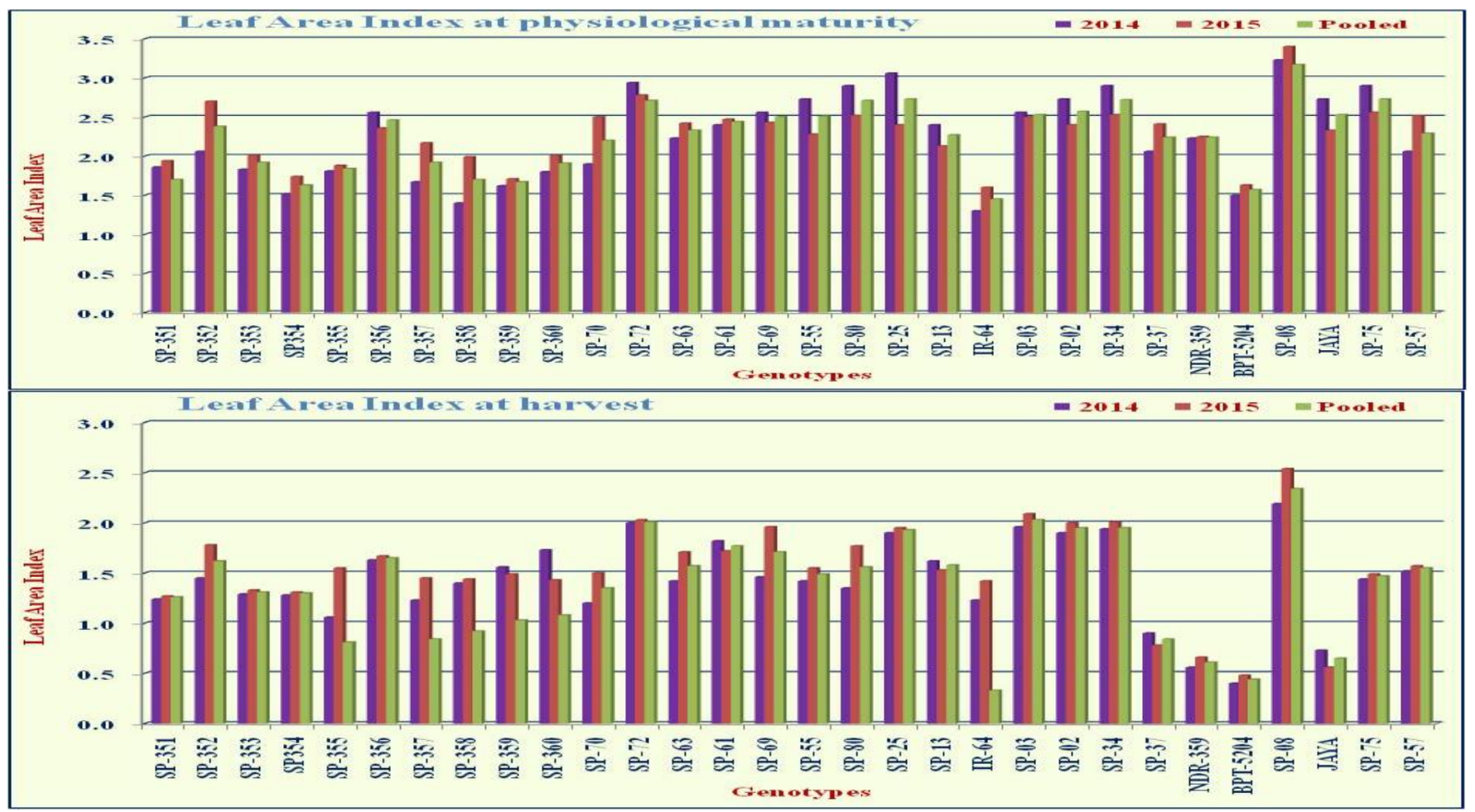


Figure.3 Leaf Area Duration (LAD) at active tillering and panicle initiation 2014-15 and pooled of advanced breeding lines of rice

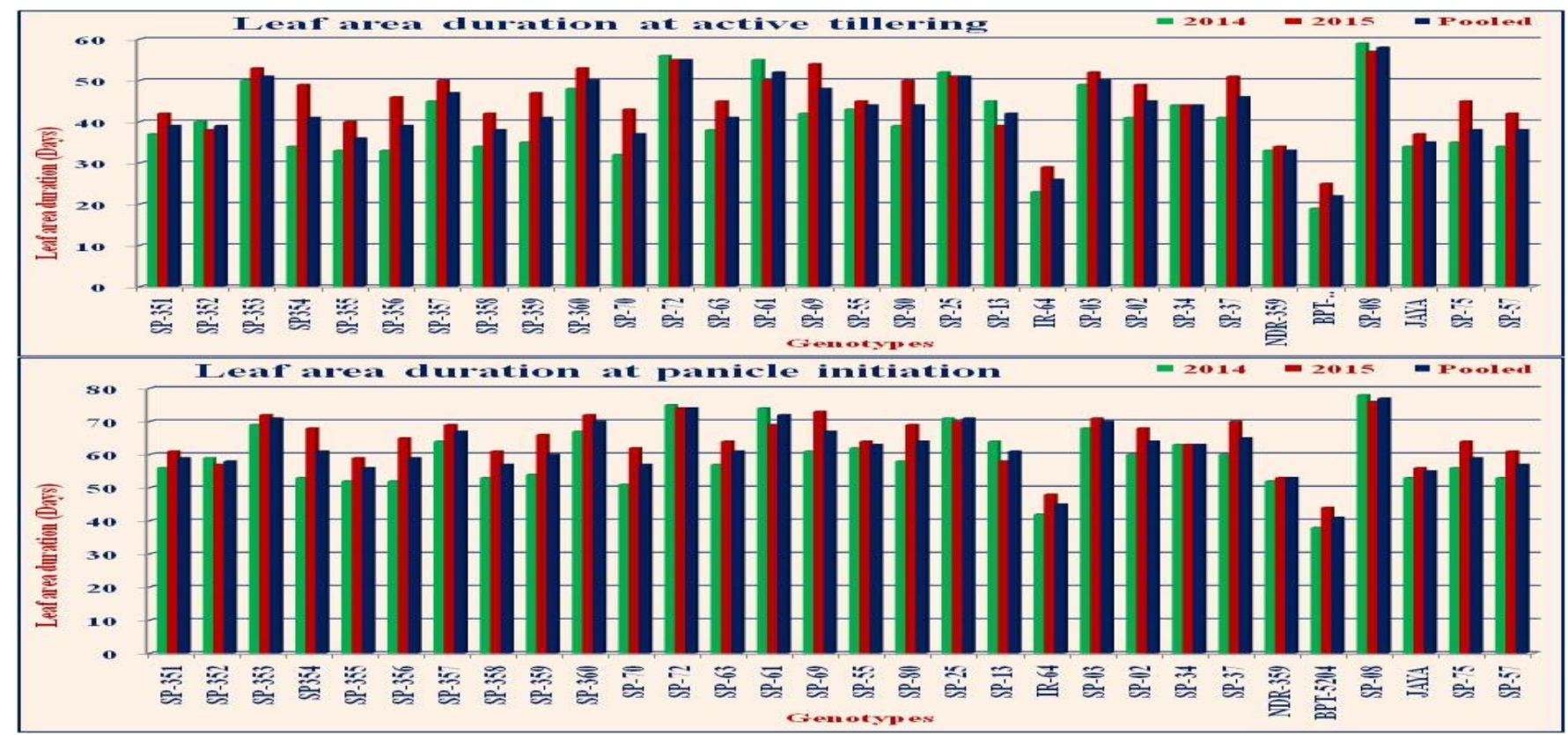

Figure.4 Leaf Area Duration (LAD) at physiological maturity and harvest 2014-15 and pooled of advanced breeding lines of rice

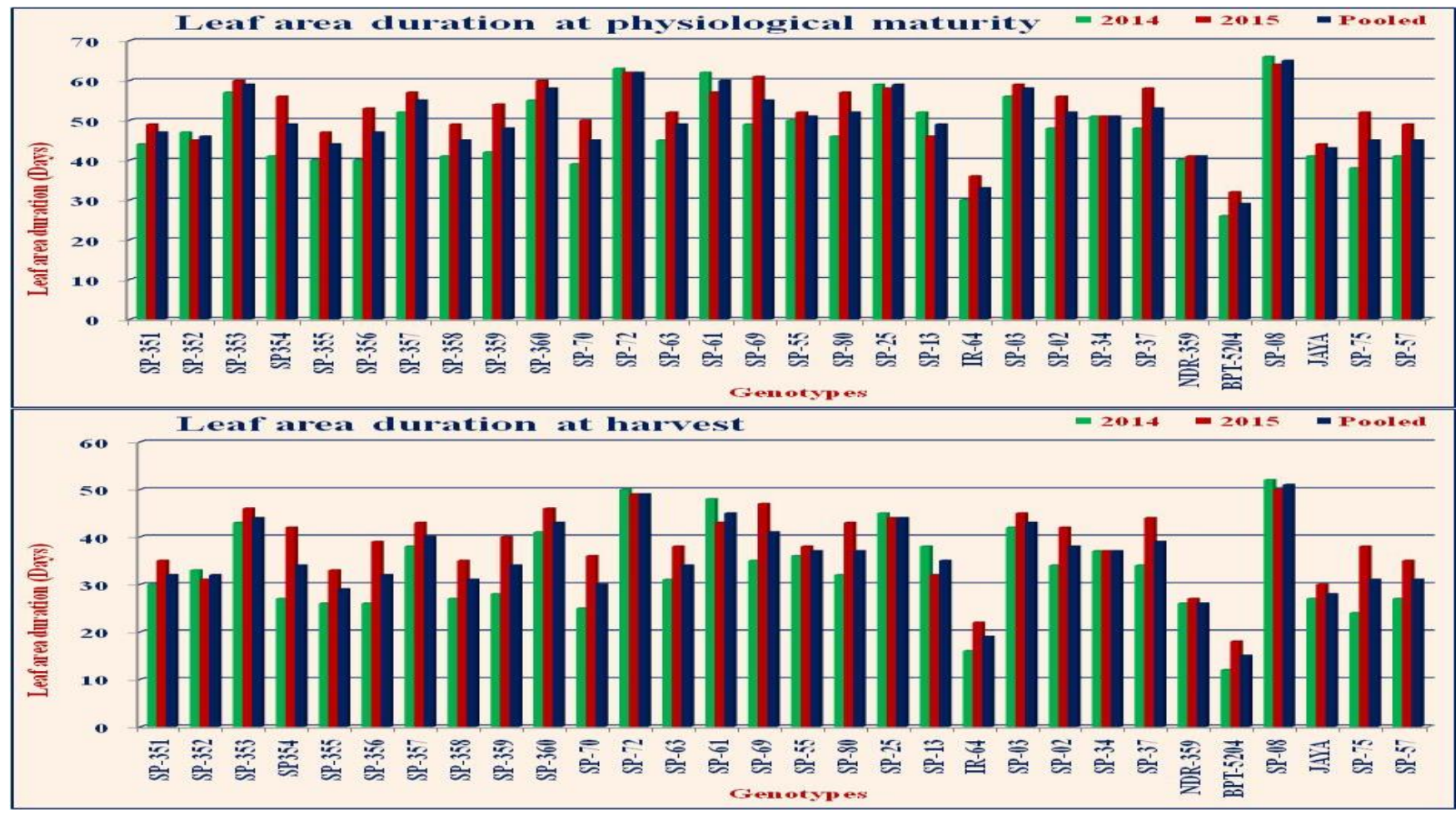


Figure.5 Crop Growth Rate (CGR) at active tillering and panicle initiation 2014, 15 and pooled of advanced breeding of rice

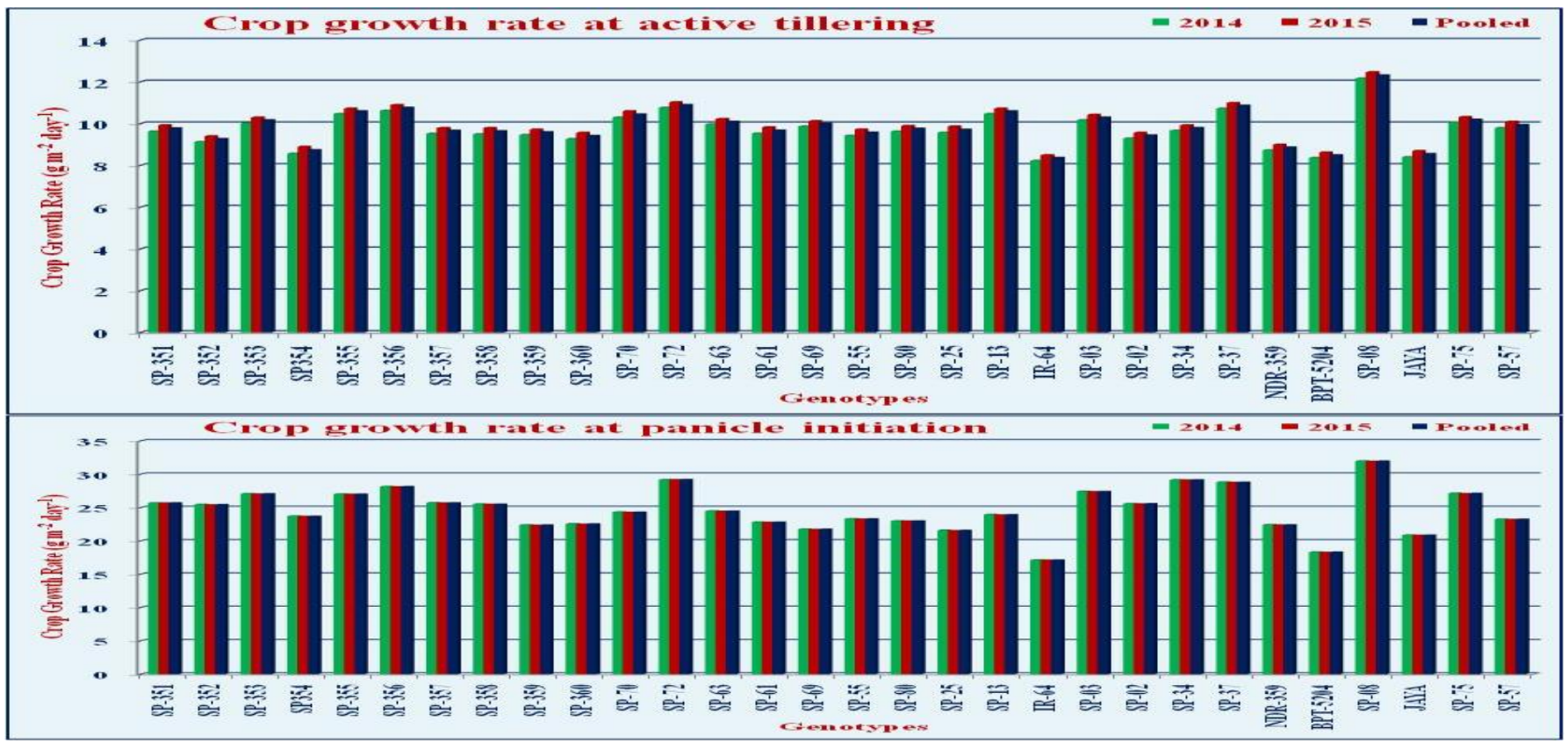

Figure.6 Crop Growth Rate (CGR) at physiological maturity and harvest 2014, 2015 and pooled of advanced breeding lines of rice

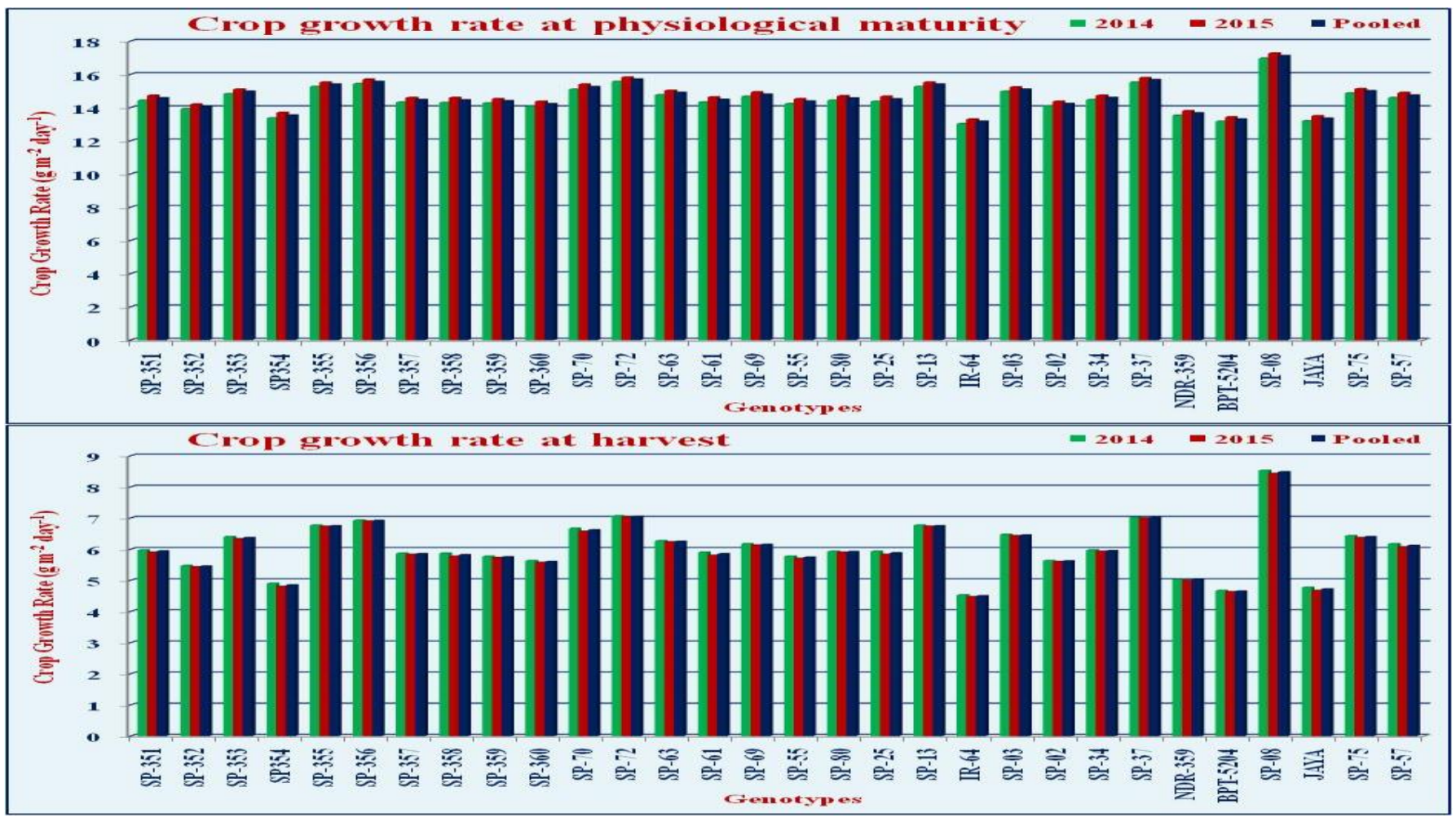


The genotype SP-08 (32.00 $\mathrm{g} \mathrm{m}^{-2}$ day $\left.^{-1}\right)$ and SP-72 (29.20 $\mathrm{g} \mathrm{m}^{-2}$ day $\left.^{-1}\right)$ showed maximum crop growth rate compared to quality check BPT-5204. While, IR-64 (17.13 $\mathrm{g} \mathrm{m}^{-2}$ day $\left.^{-1}\right)$ showed minimum during the panicle initiation stage in first season. In second season genotype SP-08 (31.93 $\mathrm{g} \mathrm{m}^{-2}$ day $\left.^{-1}\right)$ and SP-72 (29.17 $\mathrm{g} \mathrm{m}^{-2}$ day $^{-1}$ ) showed maximum value compared to quality check BPT-5204.

While, IR-64 (17.10 $\mathrm{g} \mathrm{m}^{-2}$ day $\left.^{-1}\right)$ showed the minimum values. Pooled data revealed that the genotype SP-08 (31.97 $\mathrm{g} \mathrm{m}^{-2}$ day $\left.^{-1}\right)$ and SP-72 (29.19 $\left.\mathrm{g} \mathrm{m}^{-2} \mathrm{day}^{-1}\right)$ recorded maximum crop growth rate compared to quality check BPT-5204. While, IR-64 recorded minimum $\left(17.12 \mathrm{~g} \mathrm{~m}^{-2} \mathrm{day}^{-1}\right)$ respectively.

\section{Crop Growth Rate (CGR) $\mathrm{g} \mathrm{m}^{-2}$ day $^{-1}$ at physiological maturity stage}

In the present study all the 30 genotypes showed increased trend in the crop growth rate at physiological maturity stage (Table 3 and depicted Figure 6). Significant difference was recorded among the genotypes for crop growth rate in both the seasons as well as in pooled data. The genotype SP-08 $\left(16.97 \mathrm{~g} \mathrm{~m}^{-2}\right.$ day $^{-1}$ ) and SP-72 (15.51 $\mathrm{g} \mathrm{m}^{-2}$ day $^{-1}$ ) showed maximum crop growth rate compared to quality check BPT-5204.

While, IR-64 (13.03 $\mathrm{g} \mathrm{m}^{-2}$ day $\left.^{-1}\right)$ showed minimum during the physiological maturity stage in first season. In second season genotype SP-08 (17.27 $\left.\mathrm{g} \mathrm{m}^{-2} \mathrm{day}^{-1}\right)$ and SP-72 $\left(15.83 \mathrm{~g} \mathrm{~m}^{-2}\right.$ day $\left.^{-1}\right)$ showed maximum value compared to quality check BPT-5204. While, IR-64 (13.30 $\mathrm{g} \mathrm{m}^{-2}$ day $\left.^{-1}\right)$ showed the minimum value. Pooled data revealed that the genotype SP-08 (17.12 $\left.\mathrm{g} \mathrm{m}^{-2} \mathrm{day}^{-1}\right)$ and SP-72 (15.70 $\mathrm{g} \mathrm{m}^{-2}$ day $^{-1}$ ) recorded maximum crop growth rate compared to quality check BPT5204. While, IR-64 recorded minimum (13.17 $\mathrm{g} \mathrm{m}^{-2}$ day $^{-1}$ ) respectively.

\section{Crop Growth Rate (CGR) $\mathrm{g} \mathrm{m}^{-2}$ day $^{-1}$ at harvest stage}

In the present study all the 30 genotypes showed increased trend in the crop growth rate at harvest stage (Table 3 and depicted 6). Significant difference was recorded among the genotypes for crop growth rate in both the seasons as well as in pooled data. The genotype SP-08 (8.53 $\mathrm{g} \mathrm{m}^{-2}$ day $\left.^{-1}\right)$ and SP-72 (7.07 $\mathrm{g} \mathrm{m}^{-2}$ day $^{-1}$ ) showed maximum crop growth rate compared to quality check BPT5204. While, IR-64 (4.53 $\left.\mathrm{g} \mathrm{m}^{-2} \mathrm{day}^{-1}\right)$ showed minimum during the harvest stage in first season. In second season genotype SP-08 $\left(8.43 \mathrm{~g} \mathrm{~m}^{-2} \mathrm{day}^{-1}\right)$ and SP-72 (7.03 $\mathrm{g} \mathrm{m}^{-2}$ day $^{-}$ $\left.{ }^{1}\right)$ showed maximum value compared to quality check BPT-5204. While, IR-64 (4.47 $\mathrm{g} \mathrm{m}^{-2}$ day $^{-1}$ ) showed the minimum during harvest stage. Pooled data revealed that the genotype SP-08 (8.48 $\mathrm{g} \mathrm{m}^{-2}$ day $\left.^{-1}\right)$ and SP-72 (7.05 $\mathrm{g} \mathrm{m}^{-2}$ day $^{-1}$ ) recorded maximum crop growth rate compared to quality check BPT5204. While, IR-64 recorded minimum (4.50 $\mathrm{g} \mathrm{m}^{-2}$ day $^{-1}$ ) respectively.

\section{References}

Azarpour, E., Motamed, M. K, Moraditochaee, M., Bozorgi, H. R. 2011. Effect of nitrogen fertilizer and nitroxin biofertilizer management on growth analysis and yield of rice cultivars (Iran). World Applied Sciences Journal 14(2), 193-198.

Choudhary, R. L., Kumar, D., Shivay, Y. S., Lata., Singh, G. and Singh, N. 2010. Performance of rice (Oryza sativa) hybrids grown by the system of rice intensification with plant growthpromoting rhizobacteria. Indian Journal of Agricultural Sciences. 80(10): 91720.

Kulkarni, K. P., Vishwakarma, C., Sahoo, S. P., Lima, J. M., Nath, N., Dokku, P. 2013. Phenotypic characterization and 
genetic analysis of dwarf and early flowering mutants of rice variety Nagina22. An International Journal of Rice 50, 18-25.

Kumar, M. S., Yakadri, M. and ShaikMohammad. 2009. Evaluation of genotypic response for system of rice intensification-a novel technology. Plant Archives 6(1): 329-31.

Paul, S. K., Rahman, K. S., Sarkar, M. A. R. (2013). Physiological attributes of transplant Aman rice (cv. BRRI dhan52) as affected by tiller seedlings and urea super granules. Progress. Agriculture. 24 (1\&2): 17-27.

Radford, P. J. 1967. Growth analysis formulae: Their use and abuse. Crop Science. 8: 171- 175.

Sadeghi, H., Bohrani, M. J. 2001. Effect of plant density and nitrogen rates on physiological indices of corn (zea mays). Iranian Journal of Crop Sciences. 1, 13-25.

Singh, N., Dinesh, K., Thenua, O.V.S. and Tyagi, V.K. 2009. Influence of spacing and weed management on rice varieties under system of rice intensification. Indian Journal of Agronomy 57(2): $138-42$.

Watson, D. 1952. The physiological basis of variation in yield. Advances in Agronomy. 6: 103-109.

Yoshida, S., D. A. Forno, J. H. Cock and K. A. Gomes, 1976. Laboratory Manual for physiological studies of rice. IRRI., Los Banos. Philippines.

\section{How to cite this article:}

Dev Kumar, D., D. Vishnu Vardhan Reddy, P. Raghuveer Rao, M. Sheshu Madhav, V. Gouri Shankar and Vishalakshi, B. 2019. Assessment of Diversity in Physiological Characters of Advanced Breeding Lines of Rice (Oryza sativa L). Int.J.Curr.Microbiol.App.Sci. 8(07): 22862298. doi: https://doi.org/10.20546/ijcmas.2019.807.279 